\title{
Editorial
}

Justus Haucap*

\section{Kehrtwende in der deutschen Drogenpolitik - endlich!}

https://doi.org/10.1515/pwp-2021-0061

Liebe Leserin, lieber Leser,

die neue deutsche Bundesregierung hat eine Kehrtwende in der Drogenpolitik angekündigt. „Gebt das Hanf frei“, hatte der Grünen-Politiker Hans-Christian Ströbele 2002 auf der Hanfparade in Berlin gerufen. Schon 30 Jahre zuvor hatte der liberale Ökonom Milton Friedman, der ansonsten wohl wenig mit Ströbele gemeinsam hatte, für die Legalisierung von Cannabis geworben. ${ }^{1}$ Im Jahr 2005 hatten dann über 530 amerikanische Ökonomen an den damaligen amerikanischen Präsidenten George W. Bush geschrieben, um ihn von den Vorzügen einer liberalen Drogenpolitik zu überzeugen. Neben Friedman gehörten auch die beiden Ökonomie-Nobelpreisträger George Akerlof und Vernon Smith zu den prominenten Unterzeichnern. Mittlerweile haben 18 amerikanische Bundesstaaten sowie Washington D.C. den Cannabis-Konsum für den Freizeitgebrauch legalisiert. Kanada hat 2018 die Prohibition gleich national beendet. Es sieht so aus, als könnte nun auch Deutschland als erstes Land in Europa die Legalisierung beschließen - 50 Jahre nach Friedmans Beitrag.

Die Rubrik Unsere Welt in Zahlen widmet sich daher dem Cannabis-Konsum. Wie Abbildung 1 zeigt, hat fast jeder dritte Deutsche schon einmal im Leben Cannabis konsumiert. Unter jüngeren ist diese Rate deutlich höher. Auch die sogenannte 12-Monats-Prävalenz ist in den vergangenen 30 Jahren merklich gestiegen und liegt mit 8,3 Prozent im Jahr 2018 so hoch wie nie zuvor. Die 12Monats-Prävalenz gibt an, welcher Anteil der Befragten in den zurückliegenden 12 Monaten wenigstens einmal Cannabis konsumiert hat. Die 30-Tages-Prävalenz, die sehr regelmäßige Nutzer kennzeichnet, ist hingegen im Laufe der Zeit relativ stabil geblieben. Abbildung 2 zeigt die

1 Friedman, M. (1972), Prohibition and drugs, Newsweek vom 1. Mai, S. 104.

*Kontaktperson: Justus Haucap, Düsseldorf Institute for Competition Economics (DICE), Heinrich-Heine-Universität Düsseldorf, Universitätsstr.1, 40225 Düsseldorf, E-Mail: haucap@dice.hhu.de
12-Monats-Prävalenzraten in ausgewählten Regionen der Welt im Quervergleich.

Nicht allein die rechtliche Behandlung bestimmt, wie stark der Cannabis-Konsum in einem Land verbreitet ist. In den Niederlanden ist die 12-Monats-Prävalenz höher als in Deutschland, aber geringer als im ebenfalls prohibitiven Frankreich. In den Vereinigten Staaten war und ist der Konsum durchgängig stärker verbreitet als in Europa. Selbst in Texas hat der Cannabis-Konsum trotz einer sehr repressiven Drogenpolitik deutlich zugenommen. Ganz allgemein nimmt der Cannabis-Konsum in aller Welt zu in Staaten, die den Konsum legalisiert haben, ebenso wie in Staaten, die an der Prohibition festhalten. Allerdings neigen amerikanische Bundesstaaten mit hohen Prävalenzraten eher zur Legalisierung. Von diesen Vorreitern lässt sich lernen, wie eine Marktordnung für die Cannabiswirtschaft aussehen sollte, um echten Jugend- und Verbraucherschutz zu gewährleisten und den Schwarzmarkt möglichst schnell auszutrocknen.

Aus aktuellem Anlass gratuliert Viktor Vanberg (Freiburg) Hans Albert zum 100. Geburtstag, und Karl-Heinz Paqué (Magdeburg) würdigt und gedenkt Herbert Giersch, der im Jahr 2021 ebenfalls 100 Jahre alt geworden wäre. Thomas Apolte (Münster) liefert sodann in der Rubrik Wissenschaft im Überblick einen Survey der Literatur zur politischen Ökonomie von Massenprotesten und Revolutionen. Er legt dar, unter welchen Bedingungen sich in autokratischen Regimen Massenprotest formiert, wann dieser die Stabilität eines autokratischen Regimes gefährdet und, als Ausblick, wann ein Regimekollaps den Weg in eine demokratische Gesellschaft ebnen kann.

Das Gespräch führte Karen Horn mit Christoph M. Schmidt (Essen/Bochum). Themen sind die wirtschaftspolitischen Herausforderungen für die deutsche Bundesregierung in der kommenden Legislaturperiode, Chancen und Risiken der Industriepolitik, notwendige Änderungen in der Klimapolitik sowie Arbeitsmarkt- und Sozialpolitik.

In den Beiträgen aus der Forschung erörtern zunächst Reiner Eichenberger (Fribourg), Marco Portmann (Luzern), Patricia Schafer (Fribourg) und David Stadelmann (Bayreuth), ob das Schweizer Wahlsystem mit Mehrheitswahlen in Mehrpersonenwahlkreisen ein Grund für den wirt- 
schaftlichen und gesellschaftlichen Erfolg der Schweiz ist. Sie bejahen das, denn nach ihrer Analyse gibt dieses System Politikern Anreize, sich in der Mitte des politischen Spektrums zu positionieren. In der Folge würden zumeist Kandidaten aller großen Parteien gewählt, sodass Regierungen und Parlamente parteilich stark durchmischt seien. Dies sei eine entscheidende Ursache der für die Schweiz typischen „Konkordanz“ und hohen politischen Stabilität.

Lars P. Feld (Freiburg), Lukas Nöh, Wolf Heinrich Reuter und Mustafa Yeter (alle SVR Wiesbaden) weisen im zweiten Beitrag darauf hin, dass die Wiedereinhaltung der Schuldenbremse auf Bundesebene in den kommenden Jahren eine Reduktion der im Rahmen der Corona-Pandemie stark gestiegenen Neuverschuldung in Deutschland notwendig mache. Sie stellen die fiskalpolitischen Spielräume im Vergleich mit der maximal zulässigen Nettokreditaufnahme unter der Schuldenbremse dar. Diese Spielräume erhöhten sich insbesondere durch den Abbau von Rücklagen sowie durch sinkende Zinsausgaben. Zudem passe die Konjunkturkomponente den fiskalischen Spielraum symmetrisch an die konjunkturellen Gegebenheiten an und ermögliche das uneingeschränkte Wirken der automatischen Stabilisatoren. Einschränkend wirkten die Tilgungszahlungen, die sich durch die Inanspruchnahme der Ausnahmeregelung ergeben. Die Autoren zeigen Alternativen auf, wie die Tilgungen zusammengefasst und konjunkturgerecht gestaltet werden können.
Im dritten Beitrag beleuchten Jörg Thomä und Kilian Bizer (beide Göttingen) die Forschungs- und Innovationspolitik, die auf Lernen und Innovieren nach dem Prinzip „Science-Technology-Innovation“ (STI) fußt. Gerade in Deutschland sind allerdings viele kleine und mittlere Unternehmen (KMU) in der Lage, in einem anderen Modus zu innovieren: mit „Learning by doing-Using-Interacting“ (DUI). Eine Reihe neuer Studien ermöglicht ein besseres Verständnis der Rolle, den DUI für die Innovationsfähigkeit der KMU spielt. Die Autoren erörtern deren Ergebnisse und ordnen sie ein.

Das Heft beenden wir mit einem Nachruf auf Heinrich W. Ursprung, der am 17. August 2021 kurz vor seinem 70. Geburtstag verstorben ist. Niklas Potrafke (München) und Carsten Hefeker (Siegen) würdigen Ursprungs Beiträge zur Weiterentwicklung der politischen Ökonomie wie auch seine Leistungen für den Verein für Socialpolitik, die Volkswirtschaftslehre in Deutschland und den wissenschaftlichen Nachwuchs.

Wie immer wünsche ich Ihnen den ein oder anderen Erkenntnisgewinn beim Lesen sowie eine anregende Lektüre. Für Ihr Feedback bin ich jederzeit dankbar.

Justus Haucap

Twitter: @PerspektivenWP 